\title{
Effect of Substrate Temperature on the Structural and Optical Properties of Nanocrystalline Cadmium Selenide Thin Films Prepared by Electron Beam Evaporation Technique
}

\author{
N.J. Suthan Kissinger ${ }^{a, *}, J_{\text {S Suthagar }}^{b}$, B. Saravana Kumar ${ }^{c}$, T. Balasubramaniam ${ }^{d}$ \\ AND K. PERUmal ${ }^{e}$ \\ ${ }^{a}$ Department of Physics, Loyola Institute of Technology \& Science, Tamilnadu, India \\ ${ }^{b}$ Department of Physics, Karunya University, Coimbatore - 641114, India \\ ${ }^{c}$ School of Advanced Materials Engineering,Chonbuk National University,South Korea \\ ${ }^{d}$ Department of Physics, Kongunadu College of Arts \& Science, Coimbatore, India \\ ${ }^{e}$ Department of Physics, SRMV College of Arts \& Science, Coimbatore - 641020, India
}

(Received March 22, 2010; in final form July 9, 2010)

\begin{abstract}
Cadmium selenide (CdSe) thin films on glass substrates were prepared by physical vapour deposition under vacuum using the electron beam evaporated technique for different substrate temperatures: room temperature, $100,200,300^{\circ} \mathrm{C}$, respectively. X-ray diffraction analysis indicates that the films are polycrystalline, having hexagonal (wurtzite) structure irrespective of their substrate temperature. All the films show most preferred orientation along (002) plane parallel to the substrates. The microstructural parameters such as particle size, stress, strain and dislocation density were calculated. The grain size of deposited CdSe films is small and is within the range of 18 to $42 \mathrm{~nm}$. The optical absorption spectra of electrom beam deposited CdSe films were studied in the wavelength region of 250-2500 $\mathrm{nm}$. The energy gap $\left(E_{\mathrm{g}}\right)$ values (allowed direct transitions), calculated from the absorption spectra, ranged between 1.77 and $1.92 \mathrm{eV}$. The surface morphological quality of electron beam evaporated CdSe films were analyzed by scanning electron microscopy and atomic force microscopy.
\end{abstract}

PACS numbers: 68.37.Hk, 81.05.Dz

\section{Introduction}

Binary semiconductor compounds belonging to II-VI groups of the periodic table are important due to their potential use in photoconductive devices and solar cells [1-4]. Among the II-VI group elements, CdSe is an important material for the development of various modern technologies of low cost devices such as light emitting diodes, solar cells, photodetectors, electrophotography, lasers and high-efficiency thin film transistors etc. [5-7]. The distribution of grain size and other structural and morphological properties of the films strongly affect the performance and reliability of active devices fabricated on such layers. In recent years the investigation of electrical and optical properties of CdSe thin films have been given much importance in order to improve the performances of the devices and also for finding new applica-

* corresponding author; e-mail: suthanjk@gmail.com tions [8-11]. A number of methods have been used to prepare CdSe thin films (including physical vapour deposition, sputtering, spray pyrolysis, electrodeposition, etc.) $[12-15]$. Out of the various methods available, the physical vapour deposition in its variants is often used because it offers many possibilities to modify the deposition parameters and to obtain films with controlled structures and properties. However, it is interesting to study the deposition of CdSe thin films by means of simple and inexpensive techniques and using more commercially available substrates, in view of the low cost production of CdSe devices. Among the various deposition techniques available for the preparation of CdSe thin films, the electron beam evaporation (EBE) technique is very important and promising deposition technique, because it is relatively simple, inexpensive and convenient, in particular for large area deposition. Also this method has been considered largely for the growth of device quality thin films [16-18]. The photovoltaic device performance of CdSe films basically depends on their structural, sur- 
face morphological, compositional and optical properties. It is important that the improvement of materials properties requires closer inspection of preparation conditions and also the above said properties of the films. Hence, in the present study we have investigated the structural, optical and morphological properties of electron beam evaporated CdSe thin films and the effect of substrate temperature $\left(T_{\mathrm{S}}\right)$ on these properties in a detailed manner and the results are presented.

\section{Experimental}

Thin films of CdSe were prepared by EBE technique using a HINDHI-VAC vacuum unit (model: 12A4D) fitted with electron beam power supply (model: EBG-PS$-3 \mathrm{~K}$ ). Well degreased microscopic glass plates have been employed as the substrates in the present work. Dry CdSe powder (Aldrich, 99.99\%) was shaped into pellets, taken in a graphite crucible and kept in water cooled copper hearth of the electron gun. The pelletized CdSe targets were heated by means of an electron beam collimated from the dc heated tungsten filament cathode. The surface of the CdSe pellet was bombarded by $180^{\circ}$ deflected electron beam with an accelerating voltage of $5 \mathrm{kV}$ and a power density of about $1.5 \mathrm{~kW} / \mathrm{cm}^{2}$. The evaporated species from CdSe pellet were deposited as thin films on the substrates in a pressure of about $1 \times 10^{-5}$ mbar. Each substrate was placed normal to the line of sight from the evaporation source at a polar angle to avoid shadow effects and also to obtain uniform deposition. The different preparation parameters such as source to substrate distance $(15 \mathrm{~cm})$ and partial pressure $\left(10^{-5}\right.$ mbar $)$ have been varied and optimized for depositing uniform, well adherent and transparent films. The rate of evaporation $(0.5 \mathrm{~nm} / \mathrm{s})$ was used to deposit all CdSe films to a thickness of $200 \mathrm{~nm}$. CdSe films were prepared at room temperature (RT), 100, 200 and $300^{\circ} \mathrm{C}$, respectively, to study the effect of substrate temperature on the structural, optical, and morphological properties.

The crystalline size $(D)$ is calculated using the Scherrer formula from the full-width half-maximum $(\mathrm{FWHM})(\beta)$ :

$$
D=0.94 \lambda /(\beta \cos \theta),
$$

where $\lambda$ is the wavelength of the $\mathrm{X}$-ray used, $\beta$ is the FWHM, $D$ is the particle size value and $\theta$ is half the angle between incident and the scattered X-ray beams. The strain values $(\varepsilon)$ can be evaluated by using the following relation:

$$
\varepsilon=(\lambda D \cos \theta-\beta) / \tan \theta .
$$

The lattice parameter $(a)$ is determined for hexagonal structure by the following expression:

$$
1 / d^{2}=4\left(h^{2}+h k+k^{2}\right) /\left(3 a^{2}\right)+L^{2} / c^{2},
$$

where $h, k, l$ are the Miller indices of the lattice plane. The dislocation density $(\delta)$ has been calculated by using the following formula [16] for cubic CdSe thin films:

$$
\delta=15 \varepsilon a D \text {. }
$$

The spectral normal transmittance $(T)$ was measured by UV-Vis-NIR spectrophotometer over the wavelength range of 200-2500 $\mathrm{nm}$. The calculation of absorption coefficient $\alpha$ gives a higher value of $10^{4} \mathrm{~cm}^{-1}$ near the absorption edge and in the visible region. $\alpha$ depends on the radiation energy and on the composition of the films. The absorption data were analyzed using the relation for the near edge absorption of direct band gap semiconductor films by

$$
\alpha=K\left(h \nu-E_{\mathrm{g}}\right)^{1 / 2} / h \nu .
$$

The structural properties of the films were studied by the JEOL JDX X-ray diffractometer (XRD) with $\mathrm{Cu} K_{\alpha}$ radiation $(\lambda=1.5418 \AA)$. Surface morphology of the films was studied by JEOL JSM-5610 LV (Japan) scanning electron microscope (SEM) and atomic force microscopy (AFM). The optical properties of the films were analyzed by using HITACHI-3400 UV-Vis-NIR spectrophotometer in the wavelength range $200-2500 \mathrm{~nm}$.

\section{Results and discussion}

The XRD patterns of EB evaporated CdSe films on glass substrates at room temperature and different substrate temperature are shown in Figs. 1a-d. The spectra revealed almost single peak corresponding to highly oriented CdSe layers formed by EB evaporation technique. They also confirm the polycrystalline nature of the deposited film. The peaks correspond to the hexagonal phase of CdSe film. This is supported by the fact that the bulk CdSe has a highly stable hexagonal (wurtzite) structure at temperatures ranging from room temperature to its melting point $\left(\approx 1240^{\circ} \mathrm{C}\right)[9]$. At low temper-

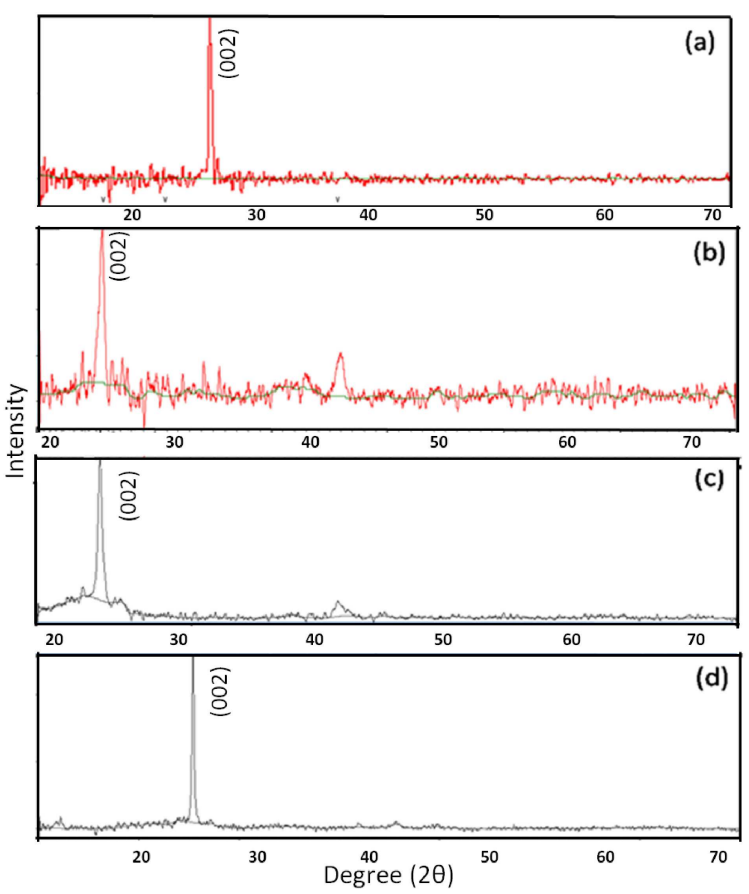

Fig. 1. X-ray diffractogram of CdSe thin films prepared on glass substrates at room temperature and at different substrate temperatures: (a) $T_{\mathrm{sub}}=\mathrm{RT}$, (b) $T_{\text {sub }}=100{ }^{\circ} \mathrm{C}$, (c) $T_{\text {sub }}=200^{\circ} \mathrm{C}$, and (d) $T_{\text {sub }}=$ $300^{\circ} \mathrm{C}$. 
atures, CdSe exists with cubic structure which is also a metastable phase. Hence, all the CdSe films deposited at various substrate temperatures ranging from room temperature to $300{ }^{\circ} \mathrm{C}$ showed only hexagonal structure in the present study. All the films have thickness of about $200 \mathrm{~nm}$. A sharp peak is observed almost in all films close to $2 \theta=25.3^{\circ}$ corresponding to (002) plane of the hexagonal phase. Another peak commonly observed is at about $2 \theta=45.9^{\circ}$ with broad and low intensity corresponding to (103) plane of hexagonal structure. The XRD pattern characteristics of CdSe films deposited at various substrate temperatures are given in Table I. The peak variation results show that at room temperature, (002) peak along with other peaks like (101), (110) and (103) are identified. When the substrate temperature is increased, the films became highly oriented along (002) direction and the other peaks are greatly suppressed. Such results have been observed for the CdSe films deposited by molecular beam epitaxy technique [19].

TABLE I

XRD patterns characteristics of CdSe films at various substrate temperatures.

\begin{tabular}{|c|c|c|c|c|c|}
\hline Substrate temp. $T_{\mathrm{s}}\left[{ }^{\circ} \mathrm{C}\right]$ & Intensity & $2 \theta\left[\left[^{\circ}\right]\right.$ observed & $d[\mathrm{~nm}]$ observed & $d[\mathrm{~nm}]$ standard & $h k l$ \\
\hline \multirow[t]{6}{*}{25} & 16 & 23.81 & 0.3718 & 0.3723 & 100 \\
\hline & 23 & 25.35 & 0.3512 & 0.3505 & 002 \\
\hline & & & & 0.3328 & 101 \\
\hline & 18 & 41.82 & 0.2138 & 0.2150 & 110 \\
\hline & 12 & 45.71 & 0.1987 & 0.1979 & 103 \\
\hline & & & & 0.1832 & 112 \\
\hline \multirow[t]{6}{*}{100} & 15 & 23.79 & 0.3719 & 0.3723 & 100 \\
\hline & 66 & 25.51 & 0.3492 & 0.3505 & 002 \\
\hline & 14 & 27.06 & 0.3272 & 0.3288 & 101 \\
\hline & & & & 0.2150 & 110 \\
\hline & 16 & & & 0.1979 & 103 \\
\hline & & & 0.1974 & 0.1832 & 112 \\
\hline \multirow[t]{6}{*}{200} & & & & 0.3723 & 100 \\
\hline & 268 & 25.47 & 0.3497 & 0.3505 & 002 \\
\hline & & & & 0.3288 & 101 \\
\hline & & & & 0.2150 & 110 \\
\hline & & & & 0.1979 & 103 \\
\hline & 24 & 45.60 & 0.1988 & 0.1832 & 112 \\
\hline \multirow[t]{6}{*}{300} & & & & 0.3723 & 100 \\
\hline & 513 & 25.39 & 0.3507 & 0.3505 & 002 \\
\hline & & & & 0.3288 & 101 \\
\hline & 4 & 41.96 & 0.2142 & 0.2150 & 110 \\
\hline & 7 & 45.71 & 0.1985 & 0.1979 & 103 \\
\hline & & & & 0.1832 & 112 \\
\hline
\end{tabular}

The effects of substrate temperature on the microstructure of CdSe films are summarized in Table II and the trend is shown in Fig. 2. The variation of the grain size of the preferred peaks with respect to substrate temperatures is shown in Fig. 2a. It shows that crystallite size of the films is increased with increasing substrate temperature. The intense and sharp peaks in XRD pattern reveal the good crystallinity of the films and also confirm the stoichiometric nature of CdSe films. It can be generally observed that strain and dislocation den- sity of the film decreases as the particle size increases which is a well-known phenomenon [20]. Strain is inherent and natural component of nanograined materials. Due to the large number of grain boundaries and the concomitant short distance between them, the intrinsic strains associated with such interfaces are always present in nanophase films. Moreover, the increasing surface energy contributes to the varying magnitude of strain. Similar results have been observed with increase of substrate temperature for vacuum evaporated CdSe films. 
Microstructural parameters of CdSe films deposited at various substrate temperatures.

\begin{tabular}{c|c|c|c|c}
\hline \hline Temperature $\left[{ }^{\circ} \mathrm{C}\right]$ & $\begin{array}{c}\text { Grain size } \\
D[\mathrm{~nm}]\end{array}$ & $\begin{array}{c}\text { Stress } \delta \\
{\left[\times 10^{14} \text { lines } / \mathrm{m}^{2}\right]}\end{array}$ & $\begin{array}{c}\text { Strain } \varepsilon \\
{\left[\times 10^{-3}\right]}\end{array}$ & $\begin{array}{c}\text { No. of crystallites } \\
{\left[\times 10^{15} \text { per unit area }\right]}\end{array}$ \\
\hline 25 & 18 & 20.81 & 1.99 & 21.09 \\
100 & 24 & 22.24 & 1.98 & 20.98 \\
200 & 36 & 18.52 & 1.74 & 11.24 \\
300 & 42 & 9.879 & 1.26 & 3.726
\end{tabular}
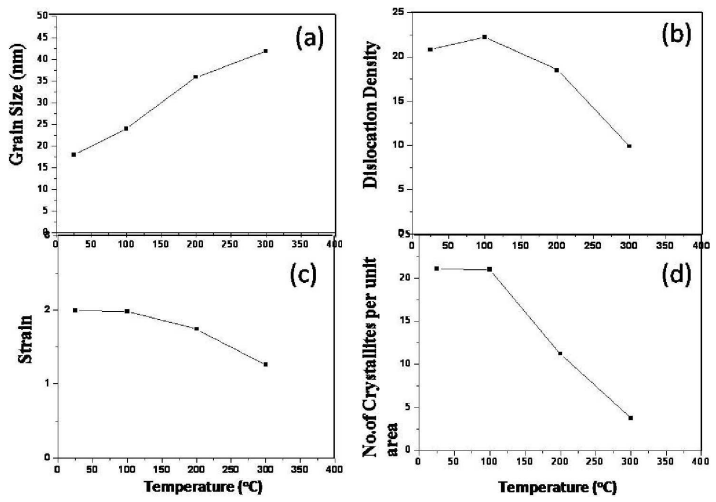

Fig. 2. Microstructural parameters: (a) grain size, (b) dislocation density, (c) strain, and (d) no. of crystallites for the CdSe thin films deposited at different substrate temperatures.

The lattice parameters values of the hexagonal structure (planes) are calculated from the equation [1-5]. The values of lattice constants for CdSe films prepared at different substrate temperatures have been listed in Table III and are shown in Fig. 3. It is inferred that the lattice parameter values are in very close agreement with the standard values. The " $c$ " values are nearly the same but may be relatively less than the standard value of $0.701 \mathrm{~nm}$ for the bulk, whereas the " $a$ " values are found to be higher as compared to the bulk value of $0.429 \mathrm{~nm}$. Such variations in lattice constants with nano grained materials can be attributed to an increased lattice strain. Such strains create local deviation of lattice constants from its bulk value which is size dependent [21]. In the present study, all the deposited CdSe films show reduced "c" values which indicate that the nanocrystallites are experiencing compression in the $a$-direction. These EB evaporated CdSe films have larger " $a$ " values indicating the action of tensile strength along $c$-direction.

This observation for our EB evaporated CdSe films are opposite to the results observed for the nanocrystalline CdSe thin films deposited by wet chemical (electrochemical) method [20]. Further, it is a fact that due to the reduced crystal size, most of the nanomaterials are found to have different crystal lattice structure when compared to the polycrystalline films or single crystalline state [22].

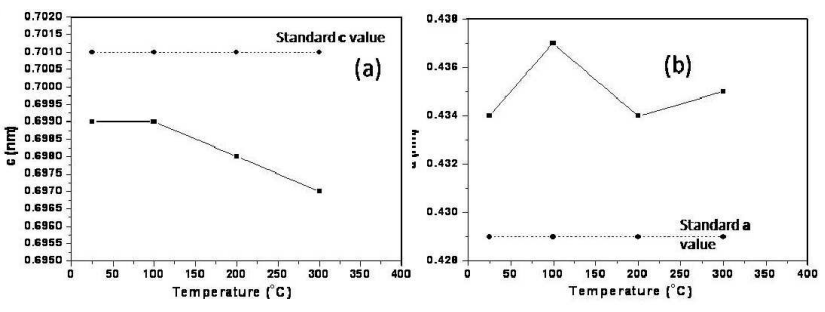

Fig. 3. The variation of lattice parameters (a) $c$ vs. $T$ and (b) $a$ vs. $T$ for the CdSe thin films deposited at different substrate temperatures.

TABLE III

Crystal lattice parameters of CdSe films deposited at various substrate temperatures.

\begin{tabular}{c|c|c|c}
\hline \hline \multirow{2}{*}{$\begin{array}{c}\text { Temperature }\left[{ }^{\circ} \mathrm{C}\right] \\
\text { CdSe }\end{array}$} & \multirow{2}{*}{$h k l$ planes } & \multicolumn{2}{|c}{ Lattice parameter } \\
\cline { 3 - 4 } & & $a[\mathrm{~nm}]$ & $c[\mathrm{~nm}]$ \\
\hline 25 & 002 & 0.434 & 0.699 \\
100 & 002 & 0.437 & 0.699 \\
200 & 002 & 0.434 & 0.698 \\
300 & 002 & 0.435 & 0.697
\end{tabular}

This is mainly attributed to the fact that the number of atoms in nanoparticle is reduced; the extra free energy associated with the surface tries to increase the fraction of particle's total free energy. It means that the smaller particle can reduce this excess free energy by changing its lattice constant, i.e., increased volume of the hexagonal crystal structure. This is affected by the contraction or expansion in either $a$ or $c$ axis. In the present studies of EB evaporated CdSe films, reduction in " $c$ " values and increase in " $a$ " values are observed and justified.

JEOL JSM-5610 LV scanning electron microscope has been used for the surface morphological analysis of CdSe films prepared at room temperature and further different substrate temperatures at 100,200 and $300^{\circ} \mathrm{C}$. The SEM pictures of RT and different substrate temperatures are shown in Figs. 4a-d. The films reveal a highly homogeneous growth up to $200{ }^{\circ} \mathrm{C}$ without pinholes and perceptible cracks and are well covered on the substrate. At $300{ }^{\circ} \mathrm{C}$, the films show few discontinuities. The particles are of nanosize and distributed all over the films matrix. 


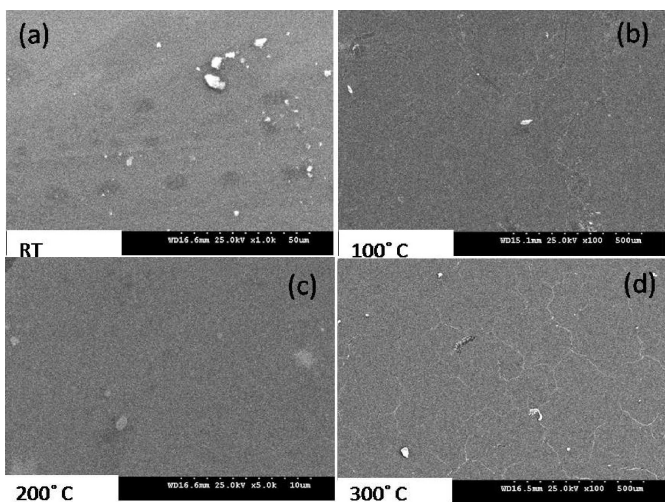

Fig. 4. SEM architecture of CdSe thin films prepared on glass substrates at room temperature and at different substrate temperatures: (a) $T_{\text {sub }}=\mathrm{RT}$, (b) $T_{\text {sub }}=$ $100^{\circ} \mathrm{C}$, (c) $T_{\text {sub }}=200^{\circ} \mathrm{C}$, and (d) $T_{\text {sub }}=300^{\circ} \mathrm{C}$.

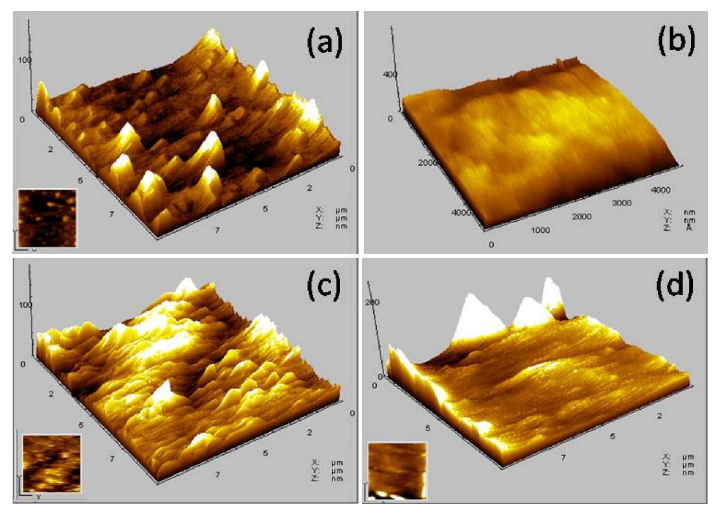

Fig. 5. AFM images of CdSe thin films prepared on glass substrates at room temperature and at different substrate temperatures: (a) $T_{\text {sub }}=\mathrm{RT}$, (b) $T_{\text {sub }}=$ $100{ }^{\circ} \mathrm{C}$, (c) $T_{\text {sub }}=200{ }^{\circ} \mathrm{C}$, and (d) $T_{\text {sub }}=300^{\circ} \mathrm{C}$.

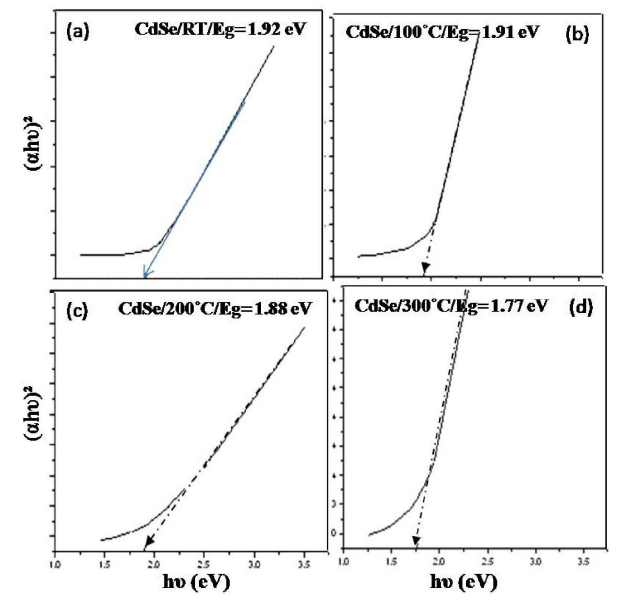

Fig. 6. $(\alpha h \nu)^{2}$ against $(h \nu)$ of CdSe thin films prepared on glass substrates at room temperature and at different substrate temperatures: (a) $T_{\text {sub }}=\mathrm{RT}$, (b) $T_{\text {sub }}=100^{\circ} \mathrm{C}$, (c) $T_{\text {sub }}=200^{\circ} \mathrm{C}$, and (d) $T_{\text {sub }}=$ $300^{\circ} \mathrm{C}$.

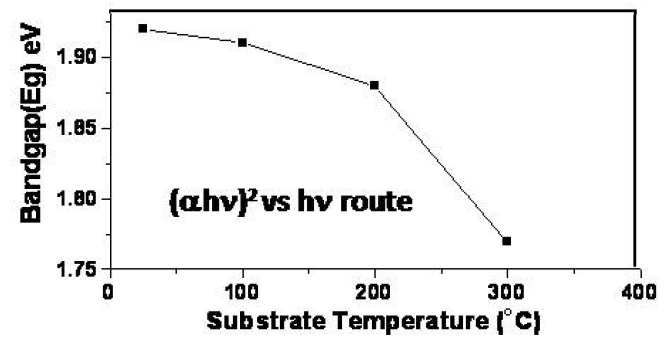

Fig. 7. Variation of $E_{\mathrm{g}}$ values for CdSe films deposited at different substrate temperatures.

Figures 5a-d represent the two-dimensional (2D) topographic AFM images of CdSe films EB evaporated onto glass substrates at RT, 100,200 , and $300^{\circ} \mathrm{C}$. The film matrix was found to have some spherical particles embedded into the background fine grained matrix. These granual particles may be due to the CdSe agglomerates deposited over the uniformly spread CdSe nanoparticles. These are observed in large number in the films deposited at RT. Our investigations showed that the grain size determined by means of AFM ranged between 100 and $200 \mathrm{~nm}$ in the matrix with the agglomerates of about $600 \mathrm{~nm}$ in size. It was found that the average size of the crystallites increases with increasing substrate temperature. The roughness of the film surface is small. Films deposited at $100^{\circ} \mathrm{C}$ show uniform surface and no agglomerates are seen. AFM images of CdSe films at 200 and $300{ }^{\circ} \mathrm{C}$ also have uniform surface but some patches and voids are present. For a detailed study on specific roughness or average roughness properties of CdSe surface and their variation with deposition temperatures, line profiles were recorded and given along with each 2D AFM pictures. This will provide valuable information on the height deviation of the roughness profile and on its lateral distribution. From the line profile analysis, the average roughness values calculated are $0.36,0.22,0.21$, and $0.23 \mathrm{~nm}$ for the CdSe films deposited at RT, 100, 200 , and $300^{\circ} \mathrm{C}$, respectively. Also they show that the vertical surface roughness deviations are higher for CdSe films deposited at RT and $300^{\circ} \mathrm{C}$ than those deposited at 100 and $200^{\circ} \mathrm{C}$. The CdSe films deposited at $100^{\circ} \mathrm{C}$ show the most uniform surface with minimum surface average roughness value. These observations show that CdSe films deposited at $100{ }^{\circ} \mathrm{C}$ have the device quality surface which will be suitable for developing photoelectrochemical (PEC) solar cells.

Figures $6 \mathrm{a}-\mathrm{d}$ show the optical transmission behavior of CdSe films deposited at RT, 100, 200, and $300^{\circ} \mathrm{C}$. All these films demonstrate good optical absorption, i.e., very small transmission below the critical wavelength of about $750 \mathrm{~nm}$. This behavior is the required optical property of the EB evaporated CdSe films prepared here which make these films suitable for photovoltaic or PEC solar cell fabrication. The values of $E_{\mathrm{g}}$ observed in Fig. 7 were found to decrease with increasing substrate temper- 
ature. The $E_{\mathrm{g}}$ values are $1.92,1.91,1.87$ and $1.77 \mathrm{eV}$ for the CdSe films deposited at temperatures RT, 100, 200, and $300{ }^{\circ} \mathrm{C}$, respectively. These values are consistent with the band gap energy values determined from the plots of $(\alpha h \nu)^{2}$ versus $h \nu$.

\section{Conclusion}

Electron beam evaporated polycrystalline CdSe thin films were prepared using quasi-closed volume technique. The structures of the films consist of fine $(18-42 \mathrm{~nm}$ average size) highly oriented grains with hexagonal (002) planes parallel to the substrate. The grain size increases when the substrate temperature increases. All the films deposited at different substrate temperature show hexagonal structure. The microstructural characterization reveals the device quality nature of these films. Optical studies show the presence of nanocrystalline particles whose size increased with temperature. A direct optical band gap of 1.77-1.92 eV was found for the investigated films. Surface morphology results show that the device quality nature of CdSe films deposited at $100{ }^{\circ} \mathrm{C}$ with a thickness of about $200 \mathrm{~nm}$ were supported by other optical and electrical data.

\section{References}

[1] P.P. Hankare, V.M. Bhuse, K.M. Garadkar, S.D. Delekar, I.S. Mulla, Semicond. Sci. Technol. 19, 70 (2004).

[2] K.R. Murali, V. Swaminathan, D.C. Trivedi, Sol. Energy Mater. Sol. Cells 81, 113 (2004).

[3] J.Y. Choi, K.J. Kim, J.B. Yoo, D. Kim, Sol. Energy 64, 41 (1998).

[4] P. O'Brien, J. McAleese, J. Mater. Chem. 8, 2309 (1998).

[5] Properties of Wide Bandgap II-VI Semiconductors, Ed. R. Bhargava, INSPEC Publications, London 1997.
[6] S. Gorer, G. Hodes, J. Phys. Chem. 98, 5338 (1994).

[7] M.T.S. Nair, P.K. Nair, R. Zingaro, E.A. Moyers, J. Appl. Phys. 74, 1879 (1993).

[8] O. Oduor, R.D. Gould, Thin Solid Films 317, 409 (1998).

[9] K.P. Mohanchandra, J. Uchil, Thin Solid Films 305, 124 (1997).

[10] M.J. Lee, Shih-Chung Lee, Solid State Electron. 43, 833 (1999).

[11] D. Samanta, B. Samanta, A.K. Chaudhuri, S. Ghorai, U. Pal, Semicond. Sci. Technol. 11, 548 (1996).

[12] K.L. Chopra, S.R. Das, Thin Film Solar Cells, Plenum Press, New York 1983.

[13] H. Cachet, R. Cortes, M. Froment, A. Etcheberry, Thin Solid Films 361-362, 84 (2000).

[14] C.M. Rouleau, D.H. Lowndes, Appl. Surf. Sci. 127-129, 418 (1998).

[15] M. Ohishi, M. Yoneta, H. Saito, H. Sawanda, S. Mori, J. Cryst. Growth 184, 57 (1998).

[16] K. Miyake, H. Kaneko, M. Sano, N. Suedomi, J. Appl. Phys. 55, 2747 (1984).

[17] N.J. Suthan Kissinger, M. Jayachandran, K. Perumal, C. Sanjeevi Raja, Bull. Mater. Sci. 30, 547 (2007).

[18] K.R. Murali, K. Sivaramamoorthy, M. Kottaisamy, S. Asath Bahadur, Physica B, Condens. Matter 404, 2449 (2009).

[19] M. Hyugaji, T. Miura, Jpn. J. Appl. Phys. 24, 950 (1985).

[20] A.S. Edelestein, R.C. Camarata, Nanomaterials: Synthesis, Properties and Application, Institute of Physics Publ., Bristol, UK 1996.

[21] M. Nirmal, D.J. Norris, M. Kuno, M.G. Bawendi, A.L. Efros, M. Roden, Phys. Rev. Lett. 75, 3728 (1995).

[22] S. Chaure, N.B. Chaure, R.K. Pandey, Physica E 28, 439 (2005).

[23] A.L. Efros, M. Roden, M. Kuno, M. Nirmal, D.J. Norris, M.G. Bawendi, Phys. Rev. B 54, 4843 (1996). 